\title{
Configuration Modeling and Experimental Verification with 3D Laser Scanning Technology for a Constrained Elastica Cable
}

\author{
Hongwang Du, Wei Xiong*, Haitao Wang, Bin Yuan and Zuwen Wang \\ Ship Electromechanical Equipment Institute \\ Dalian Maritime University \\ Dalian 116026, China \\ xiongwei@dlmu.edu.cn
}

\begin{abstract}
For large complex electromechanical products, cable digital design has a problem of low accuracy because of its large deformable characteristics. To solve the problem, an efficacious method is proposed to do cable geometry physical modeling, simulation and test for two-end constrained spatial flexible cables. Euler angles are used as generalized parametric variables to describe cable configuration on the base of Kirchhoff elastic rod theory. Via establishment of a cylindrical coordinate system according to Saint-Venant principle using end constraint, Euler angles' Analytical solutions of the Kirchhoff equations are gained. Based on an open source geometry kernel system and using numerical integration ideas, a cable configuration simulation platform is developed in $C++$ environments. Then, a cable geometry measurement bench is founded with the use of $3 D$ laser scan technology. Finally, a cable configuration simulation and experimental example is given to verify the accuracy of the proposed model and relative algorithm, through $3 D$ positional deviation analysis.
\end{abstract}

Keywords: cable configuration, Kirchhoff theory, cylindrical coordinates, 3D laser scan, open source geometry kernel

\section{Introduction}

With the development of computer graphics technology, 3D design for electromechanical products could be basically realized in a CAD modeling software, which is gradually replacing the traditional design concept of $2 \mathrm{D}$ drawing. The method is accurate for rigid object modeling, but is very difficult to realize flexible part design like cable and tube because of their complex deformable characteristics. Currently, in main 3D modeling software, a cable digital model is expressed by curvature radius through defining cable assembly key points. Obviously, the idea does not consider cable physical characteristics, and will cause large error on the contrary of the cable actual size. What's more, this rigid cable model could not simulate assembly process to guide actual assembly [1].

Early physical modeling methods for one-dimensional objects mainly contain the spline curve method, the mass-spring method and the FEM [2]. The former two are essentially simplified ideas; they only reflect flexible appearance of objects, so if the two are used as cable digital design method, larger error will appear. What was worse, the two could not describe boundary force information for constrained cables. The FEM is very accurate, but its complex calculation process could not satisfy the need of real-time for a digital assembly system. 
Kirchhoff theory was used to analyze elastic rod equilibrium early in 1859 according to the idea of kinetic analogy [3]. However, because of narrow application fields and the complex solution process, the method did not be paid attention to until the development of DNA molecular biology. Then many scholars used the method to do one-dimension object modeling. There are two difficulties for using this method to do cable physical modeling, one is how to get identified model for different boundary conditions and the other is the solving method. By now, there is analytical method and numerical method. The former uses Euler angles as variables to get the change law along the arc coordinate $[4,5]$ while the latter uses Euler parameters to discrete a cable to ensure the minimum potential energy of all parts [6,7]. In fact the two solving methods have certain simplification or hypothesis, so the model also contains certain error but few experiments have been done to verify solving methods in references. V.G.A. Goss only gave experiments on snap buckling, hysteresis and loop formation in twisted cables and the results were compared to calculations based on the Kirchhoff theory, which was obviously one-sided [8]. J. H. Liu used two binocular vision cameras to get the cable spatial geometry, but the results only reflected whether the centerline of a cable could be matched or not [7].

In this paper, to solve the problem of sidedness of experiment verification for cables, a reverse engineering method based on the 3D laser scan technology is presented, which mainly includes the following aspects.

Firstly, for a spatial two-end constrained cable, the analytical formula describing configuration is gained, with the form of cylindrical coordinates. Secondly, a cable configuration simulation platform is developed on the base of an open source geometry kernel system. Finally, an articulated arm 3D laser scanner is used to get actual cable configuration and the algorithm could be verified via comparative analysis between the simulation and the experiment.

\section{Analytical Solution of Kirchhoff Equations}

\subsection{Cable Geometry and Solution of Euler Angles}

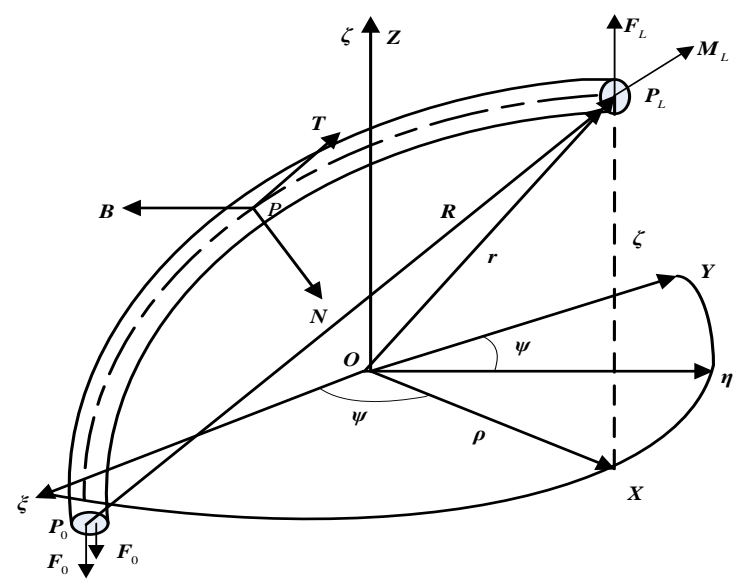

Figure 1. Geometry Description of a Spatial Deformable Cable

According to references [3, 4], cable geometry configuration could be reflected by translation and rotation of the cable cross-section along the centerline, shown as Figure 1. A fixed reference system $\mathrm{O}-\xi \eta \zeta$ and the Frenet system of cable cross-section P-NBT are 
established. Then the cable configuration could be reflected by relative rotation between the two systems. Any point position of the centerline is described by a radius vector $r(s)$, where $s$ is the arc coordinate.

Considering Euler angles $(\phi, \theta, \varphi)$ as parametric variables to describe the rotation relationship of the two reference system and neglecting volume and contact force, Kirchhoff equations for a circular cross-section cable could be gained shown as the following, where $\mathrm{A}$ and $\mathrm{C}$ is the cable bending stiffness and twisting stiffness and $F$ is the external force at the end.

$$
\left\{\begin{array}{l}
A\left[\frac{\mathrm{d}^{2} \theta}{\mathrm{d} s^{2}}-\left(\frac{\mathrm{d} \phi}{\mathrm{d} s}\right)^{2} \cos \theta \sin \theta\right]+C \frac{\mathrm{d} \phi}{\mathrm{d} s} \sin \theta\left(\frac{\mathrm{d} \varphi}{\mathrm{d} s}+\frac{\mathrm{d} \phi}{\mathrm{d} s} \cos \theta\right)-F \sin \theta=0 \\
A\left[\frac{\mathrm{d}^{2} \phi}{\mathrm{d} s^{2}} \sin \theta+2 \frac{\mathrm{d} \phi}{\mathrm{d} s} \frac{\mathrm{d} \theta}{\mathrm{d} s} \cos \theta\right]-C \frac{\mathrm{d} \theta}{\mathrm{d} s}\left(\frac{\mathrm{d} \varphi}{\mathrm{d} s}+\frac{\mathrm{d} \phi}{\mathrm{d} s} \cos \theta\right)=0 \\
C \frac{\mathrm{d}}{\mathrm{d} s}\left(\frac{\mathrm{d} \varphi}{\mathrm{d} s}+\frac{\mathrm{d} \phi}{\mathrm{d} s} \cos \theta\right)=0
\end{array}\right.
$$

Considering the linear constitutive relationship for cable material, the above equations could be integrable and the analytical solution is shown in the formula (2). Correctness of the results could be verified by any mathematical calculation software such as Maple or Mathematica.

$$
\left\{\begin{array}{l}
\gamma=\cos \theta(s)=\gamma_{1}+\left(\gamma_{2}-\gamma_{1}\right) \text { JacobiSN }^{2}\left[\frac{F}{2 A}\left(\gamma_{3}-\gamma_{1}\right)\left(s-s_{0}\right), \sqrt{\frac{\gamma_{2}-\gamma_{1}}{\gamma_{3}-\gamma_{1}}}\right] \\
\frac{\mathrm{d} \phi}{\mathrm{d} s}=\frac{M_{\zeta}-M_{3} \cos \theta}{A \sin ^{2} \theta} \\
\frac{\mathrm{d} \varphi}{\mathrm{d} s}=\frac{M_{3}}{C}-\frac{\left(M_{\zeta}-M_{3} \cos \theta\right) \cos \theta}{A \sin ^{2} \theta}
\end{array}\right.
$$

In the formula, $M_{\zeta}$ is $\zeta$-axis projection of the external torque $\boldsymbol{M}$, and $M_{3}$ is T-axis projection of $\boldsymbol{M}$. JacobiSN is the first class elliptic function, $\gamma_{i}(i=1,2,3)$ is three roots of the following equation (3) and $s_{0}$ is the arc coordinate when $\gamma=\gamma_{1}$.

$$
f(\gamma)=(h-\gamma)\left(1-\gamma^{2}\right)-\left(M_{\zeta}-M_{3} \gamma\right)^{2}
$$

In summary, as long as the tension and Euler angles and their derivative initial values are given, Euler angles' variation law along the arc coordinate could be integrated so as to gain spatial configuration of cables.

\subsection{Analytic Integral in the Cylindrical Coordinate System}

To get cable geometry configuration, it is necessary to calculate the coordinates in the fixed reference system according to the Euler angle change law along the arc coordinate. In references $[3,4]$, the coordinates $(\xi, \eta, \zeta)$ is got by rotation matrix of the two reference systems. Unfortunately, the calculation process is very complex, even mathematical calculation software could not realize but detailed calculation process did not be expressed in the two references. To solve this problem, this paper uses cylindrical coordinates as the fixed reference system to take the place of Cartesian coordinates, which is shown in Figure 1. 
For a two-end constrained cable, the direction of the external force is as the $\xi$ and three cylindrical coordinates are $(\rho, \Psi, \zeta)$, composing the system $O-X Y Z$. It is known that the radius vector could be defined as $\boldsymbol{r}(s)=(\boldsymbol{F} \times \boldsymbol{M}) / \mathrm{F}^{2}$ according to the SaintVenant principle in Elasticity. Therefore, considering the relationship between the cylindrical coordinates and the Cartesian coordinates and using cylindrical coordinates in Kirchhoff equations, the analytical formula of the three cylindrical coordinates could be gained from equations (1-3), which is shown as the following.

$$
\left\{\begin{array}{l}
\rho(s)=\frac{1}{F}\left(h A+M_{3}^{2}-M_{\zeta}^{2}-F \cos \theta\right)^{1 / 2} \\
\frac{d \psi}{d s}=\left(\frac{M_{\zeta}-M_{3} \cos \theta}{A^{2} h+M_{3}^{2}-M_{\zeta}^{2}-2 A F \cos \theta}\right) F \\
\frac{d \zeta}{d s}=\cos \theta
\end{array}\right.
$$

From formula (4), it is known that the three cylindrical coordinates could be easily integrated as long as $\theta(\mathrm{s})$ is confirmed according to boundary and initial conditions, so that cable configuration could be realized.

\section{Cable Configuration Simulation}

To simulate 3D configuration of a spatial cable based on the above model, an open source geometry kernel named Open Cascade (OCC) is used in C++ environments. OCC is a geometric modeling software platform developed by Matra Datavision Company of France and its nature is an object-oriented $\mathrm{C}++$ class library which supplies $2 \mathrm{D} / 3 \mathrm{D}$ object geometry generation, display and analysis. The cable configuration simulation platform is shown as Figure 2. In the platform, the most important function is to do spatial fixed cable configuration drawing besides basic functions of 3D geometry display and operation and could be exported in the form of common *.iges or *.step, which could be imported to any 3D CAD software.

In the whole simulation process, firstly parameters of the equation (4) is calculated according to given boundary and initial conditions, and secondly the three cylindrical coordinates describing cable spatial position is gained by using Gauss-Legendre quadrature formula. After getting series of points of cable centerline, the spatial configuration could be realized by a one-dimensional tube-like scanning generating function supplied by OCC, named CTopologyPrimitivesDoc::OnPipe(). The actual design process of the OCC class function is shown in Figure 3.

The class function firstly reads discrete cable position coordinates, then uses B-spline to render the cable centerline and finally uses a circular cross-section to scan and envelop along the centerline to get 3D cable model. 


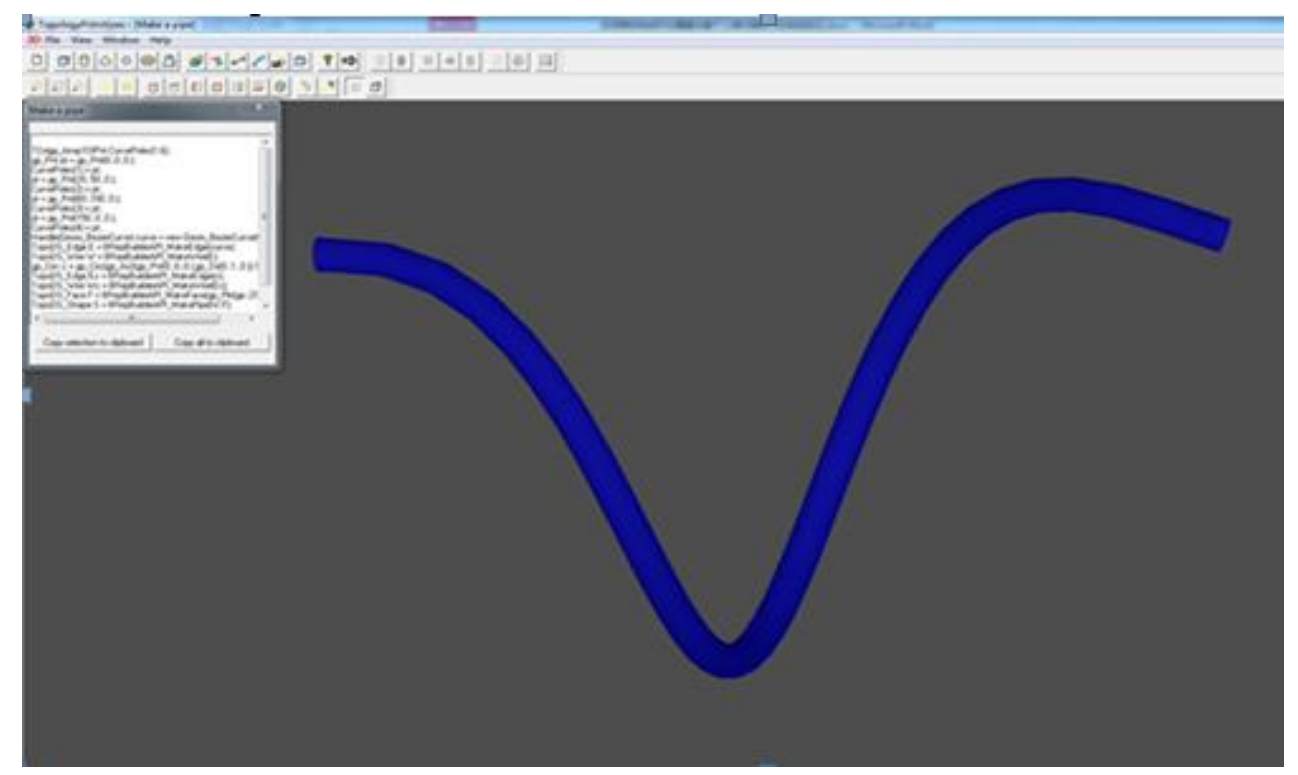

Figure 2. Cable Configuration Simulation Platform

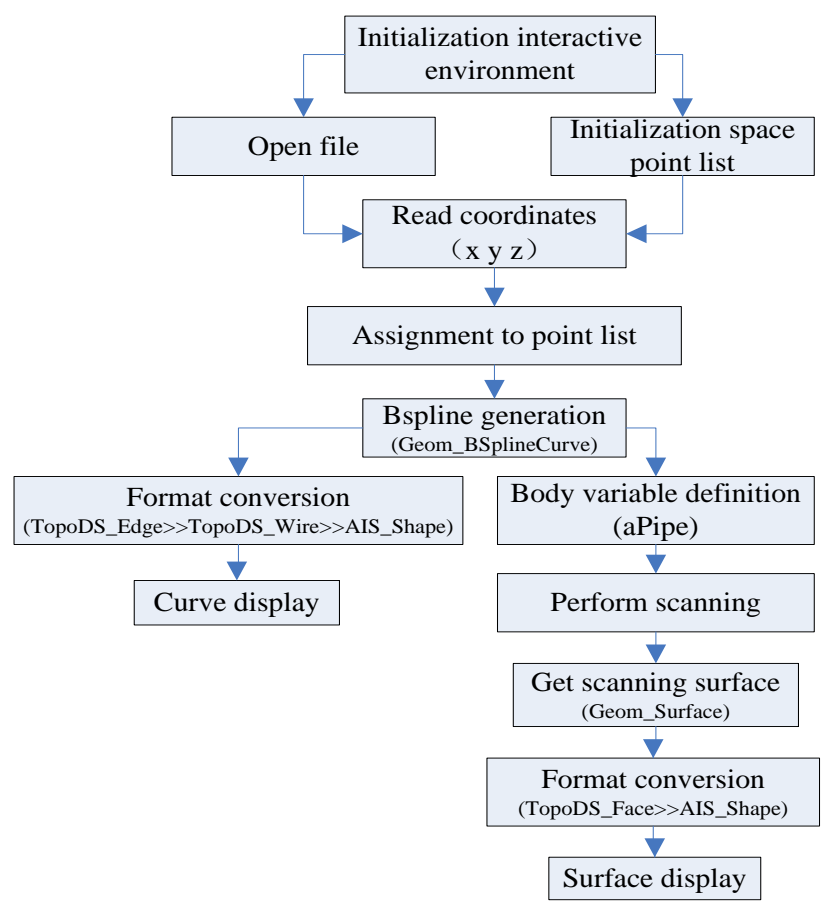

Figure 3. 3D Rendering Process of a Spatial Cable

\section{Cable Configuration Experiment Test}

To verify the correctness of the above model and calculation algorithm, a cable configuration test experimental platform is established based on the 3D laser scan technology and the platform structure is shown in Figure 4.

The measurement bench is mainly consisted of an aluminum base, support components, an articulated arm laser scanner, two torque instruments and a PC. A fixed 
reference system $\mathrm{O}-\mathrm{xyz}$ is founded. For this bench, the cable-gripped support components structure likes a series articulated robot which contains bars and gimbals, so as to make one end of a cable is fixed while the other could realize three displacements and two rotations along $\mathrm{x}$-axis and $\mathrm{y}$-axis. The movement incremental value could be got directly from graduation in the bars, so as to describe any case of a two-end constrained cable. It is assumed that the initial relative vector of the two ends is $\boldsymbol{R}_{0}$ while the changed vector is $\boldsymbol{R}_{1}$. The relationship of the two vectors is shown in the following formula.

$$
\boldsymbol{R}_{1}=\boldsymbol{R}_{0}+\left(\begin{array}{c}
\Delta x+b \cos \beta \\
\Delta y-a \cos \alpha \\
\Delta z+a \sin \alpha+b \sin \beta
\end{array}\right)
$$

In the above formula, $\Delta x, \Delta y, \Delta z$ are three changed displacement value, $\mathrm{a}$ and $\mathrm{b}$ are the length of the two rotation arm relative to $\mathrm{x}$-axis and $\mathrm{y}$-axis while $\alpha, \beta$ are the changed rotation value. After position condition is given, the constraint condition is also confirmed. Neglecting distributed force, two torque instruments could give the two-end external torque and the cable cross-section force could be calculated by the equilibrium formula (6).

$$
\left\{\begin{array}{c}
\boldsymbol{F}_{0}=-\boldsymbol{F}_{L}=\boldsymbol{F} \\
\boldsymbol{M}_{0}+\boldsymbol{M}_{L}+\boldsymbol{R} \times \boldsymbol{F}_{L}=0
\end{array}\right.
$$

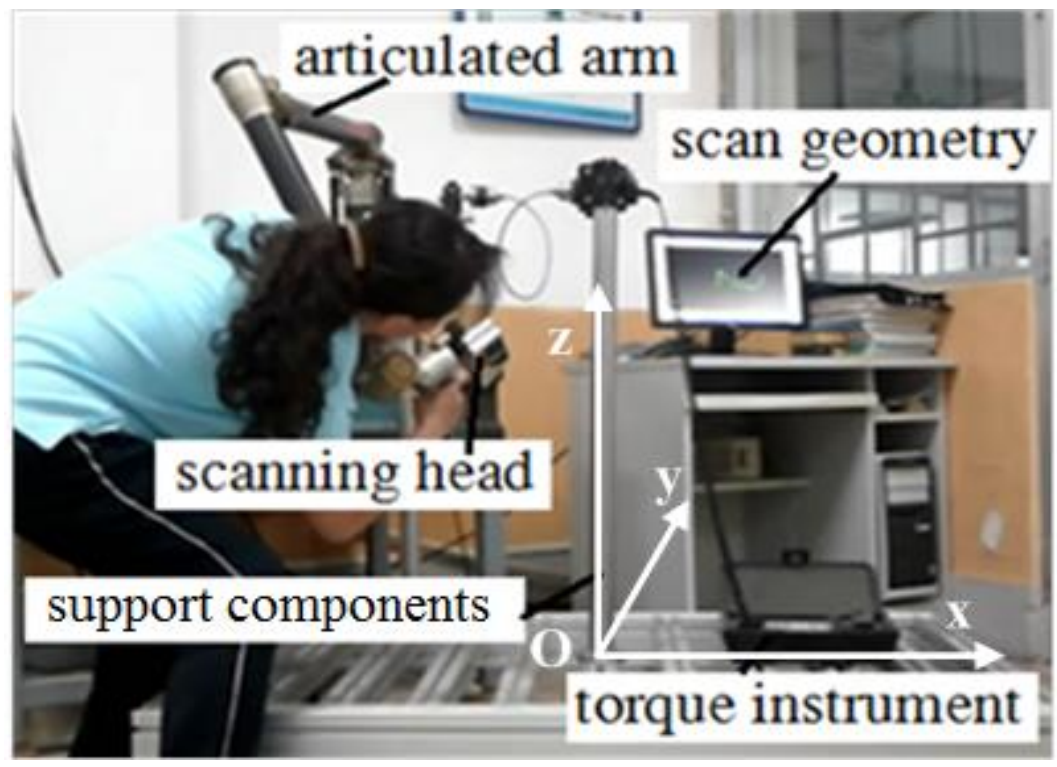

Figure 4. Cable Configuration Test Experimental Platform

Where, $\boldsymbol{R}$ is relative position vector of cable two-ends. After all the above conditions are given, actual cable configuration point cloud data could be gained by the laser scanner and matched reverse acquisition software. What is more, the cloud data and simulated $3 \mathrm{D}$ model could be imported to a reverse dealing software at the same time to finish the cable configuration error analysis.

To verify the analytical solution and algorithm in the former two parts, next a detailed example is given and the cable relative parameters are shown in Table 1. 
Table 1. Deformable Simulation Parameters

\begin{tabular}{|c|c|}
\hline Parameter name & Value \\
\hline Starting external torque, N. ${ }^{2}$ & $\left(0,0,4.75 \times 10^{-3}\right)$ \\
\hline End external torque, N. ${ }^{2}$ & $\left(0,9.5 \times 10^{-3}, 0\right)$ \\
\hline Young's module, N/m ${ }^{2}$ & $3.89 \times 10^{9}$ \\
\hline Passion ratio & 0.25 \\
\hline Diameter, mm & 4 \\
\hline Length, mm & 350 \\
\hline Position vector, mm & $(174.78,81.36,44.39)$ \\
\hline
\end{tabular}

Then two 3D modes from cable simulation and experimental measurement are both imported into the reverse engineering software Geomagic Studio, where the analysis of the two models could be done shown in Figure 5.

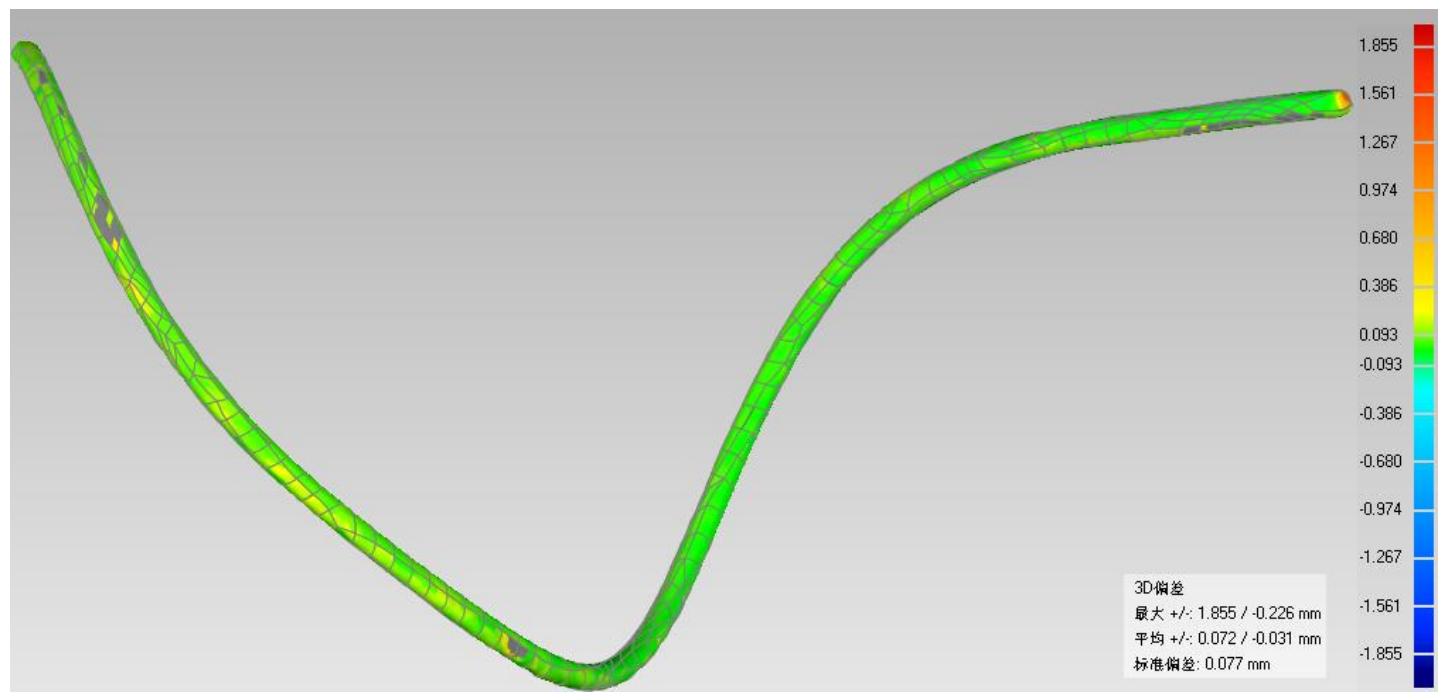

Figure 5. Cable Configuration Analytical Results

Figure 5 gives an analytical chromatogram about cable spatial position and results reflect 3D error between simulated configuration and experimental configuration under the same boundary conditions. 3D error refers to the shortest distance from any point of the simulated model to the actual model. From the Figure, it is also known larger errors appear at both ends. The reason is that cable material may appear plastic deformation because the cable is gripped at the two ends, which causes initial Euler angles are too difficult to be given very accurately. The absolute value of the largest error is $1.855 \mathrm{~mm}$ and the largest average error is $0.072 \mathrm{~mm}$. What's more, the standard error is $0.077 \mathrm{~mm}$; the standard error reflects root mean square error of all errors with normal distribution, which is only $0.2 \%$ of the cable length and $1.9 \%$ of the cable diameter. The error is enough to satisfy the needs of cable digital design and cable routing, so as to verify the model and algorithm of this paper is efficient and accurate. 


\section{Conclusions}

In this paper, an efficacious method is proposed to do cable geometry physical modeling, simulation and test for two-end constrained spatial flexible cable. The main conclusions are as the following.

Analytical solution formulas in the form of elliptic functions are gained to describe spatial cable configuration in a cylindrical reference system, the system is established according to Saint-Venant principle;

Based on the open source geometry kernel system OCC and using Gauss-Legendre quadrature formula, a cable configuration design platform is developed which could get cable 3D digital model in the form of common *.iges or *.step;

Under the same given boundary condition with simulation, cable actual configuration is measured through 3D laser scan technology. On the contrary of the two models, the standard error is only $0.077 \mathrm{~mm}$, which is only $0.2 \%$ of the cable length and $1.9 \%$ of the cable diameter, so as to verify the model and algorithm of this paper is efficient and accurate.

In the future, much more complex boundary conditions for cable modeling will be considered, such as cable is constrained by surface or clamps.

\section{Acknowledgement}

The work in this paper is supported by the National Natural Science Foundation of China (51175053) and the Fundamental Research Funds for the Central Universities (3132013017253) and the Department of Transportation Construction Projects (2013328225080).

\section{References}

[1] B. L. Wan, "Research on Cable Harness Wiring Design and Assembly Process Planning Technology in Virtual Environment”, Beijing of China: Beijing, Institute of Technology, (2007).

[2] P. J. Xia, M. L. Antonio and T. R. Maria, "A Review of Virtual Reality and Haptics for Product Assembly: from Rigid Parts to Soft Cables", Assembly Automation (S0144-5154), vol. 33, no. 2, (2013), pp. 157-164.

[3] M. Nizette, et al., "Towards a classification of Euler-Kirchhoff filaments", Journal of Mathematical Physics, vol. 40, (1999), pp. 2830-2866.

[4] S. Liu and W. Andreas, "A Symbolic-Numeric Approach to Tube Modeling in CAD Systems", Lecture Notes in Computer Science (S0302-9743), vol. 4194, (2006), pp. 279-283.

[5] G. M. Scarpello and D. Ritelli, "Elliptic integral solutions of spatial elastica of a thin straight rod bent under concentrated terminal forces", Meccanica, vol. 41, (2006), pp. 519-527.

[6] J. Spillmannn and T. M. C. Nets, IEEE Transactions on Visualization and Computer Graphics (S10772626), vol. 15 , no. 2, (2009), pp. 325-338.

[7] J. H. Liu, etc., "Motional cable harness physical characteristic oriented modeling and kinetic simulation technology in virtual environment”, Journal of Mechanical Engineering (S05776686), vol. 47, no. 9, (2011), pp. 117-124.

[8] V. G. A. Goss, G. H. M. Van der Heijden, J. M. T. Thompson and S. Neukirch, "Experiments on snap buckling, hysteresis and loop formation in twisted rods", Experimental Mechanics, vol. 45, (2005), pp. 10111.

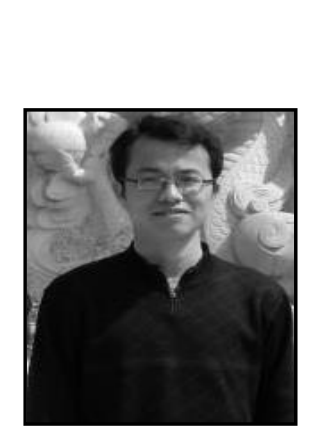

\section{Author}

Hongwang Du, Male, Ph. D. His research topic is on force feedback technology and flexible body modeling and simulation. 